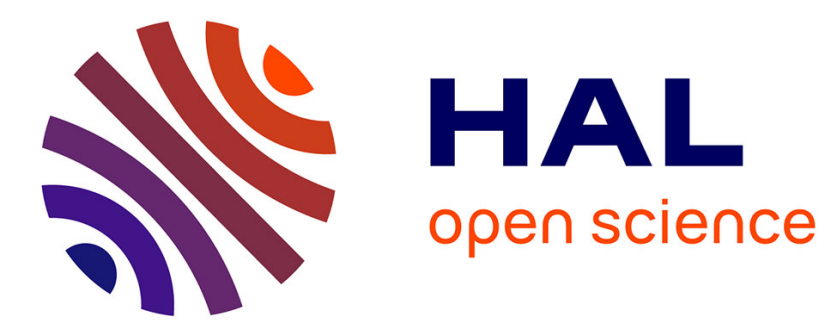

\title{
Micromechanical modelling of suffusion: towards the stability analysis of hydraulic structures.
}

\author{
A. Wautier, François Nicot, Stéphane Bonelli
}

\section{To cite this version:}

A. Wautier, François Nicot, Stéphane Bonelli. Micromechanical modelling of suffusion: towards the stability analysis of hydraulic structures.. 11th International Workshop on Bifurcation and Degradation in Geomaterials (IWBDG2017), May 2017, Limassol, Cyprus. pp.169-175, 10.1007/978-3-31956397-8_22. hal-01901761

\section{HAL Id: hal-01901761 \\ https://hal.science/hal-01901761}

Submitted on 23 Oct 2018

HAL is a multi-disciplinary open access archive for the deposit and dissemination of scientific research documents, whether they are published or not. The documents may come from teaching and research institutions in France or abroad, or from public or private research centers.
L'archive ouverte pluridisciplinaire HAL, est destinée au dépôt et à la diffusion de documents scientifiques de niveau recherche, publiés ou non, émanant des établissements d'enseignement et de recherche français ou étrangers, des laboratoires publics ou privés. 


\title{
Micromechanical modelling of suffusion: towards the stability analysis of hydraulic structures
}

\author{
Antoine Wautier, Stéphane Bonelli and François Nicot
}

\begin{abstract}
Suffusion is the most controversial of the process of internal erosion and piping as there is a lack of microscopic understanding of the degradation processes at stake in saturated soils subjected to an internal flow. Thanks to the use of a DEMPFV (Discrete Element Method - Pore-scale Finite Volume) approach, this study addresses the fully coupled problem of the suffusion of a non cohesive granular material. Eligible particles to grain detachment and grain transport are characterized from a micromechanical point of view thanks to the use of an enhanced force chain definition and the definition of a pore network. A Representative Elementary Volume with respect to the grain detachment and grain transport is then quantified.
\end{abstract}

\section{Introduction}

In various circumstances, permeable soils are subjected to internal flows which may modify their microstructure and by consequence their overall hydraulic and mechanical properties. At the microscale, this process consists in a rearrangement of particles driven by three elementary mechanisms, namely the detachment of grains from the granular skeleton, their transport through the pore network and possibly their reattachment to the granular skeleton farther away. At a larger scale, this process results in the selective erosion of the smallest particles of a soil and is referred to as suffusion within the geomechanics community [1].

Many criteria where proposed in order to assess the internal stability of a particular soil with respect to suffusion. However, there is still a lack of global approaches able to assess suffusion susceptibility by considering in the meantime the soil microstructure, its stress state and the hydraulic loading. To this respect, this paper investigates the ability of a coupled DEM-PFV approach [2] to model the effect

Antoine Wautier

AgroParisTech-ENGREF, 19 avenue du Maine, 75732 Paris, France.

Irstea UR RECOVER, 3275 Rte Cézanne, CS 40061, 13182 Aix-en-Provence Cedex 5, France.

Université Grenoble Alpes, Irstea, UR ETGR, 2 rue de la Papeterie-BP 76, F-38402 St-Martin-

d'Hères, France. e-mail: antoine.wautier@irstea.fr

Stéphane Bonelli

Irstea UR RECOVER, 3275 Rte Cézanne, CS 40061, 13182 Aix-en-Provence Cedex 5, France.

François Nicot

Université Grenoble Alpes, Irstea, UR ETGR, 2 rue de la Papeterie-BP 76, F-38402 St-Martin-

d'Hères, France. 
of an internal flow on cohesionless soils modelled as a poly-dispersed assembly of spheres. Thanks to the use of micromechanical tools based on the identification of chained particles [6] combined with the computation of autocorrelation functions [4] and the description of the pore space in terms of an oriented graph composed of pores and constrictions [7], the occurrence of the elementary mechanisms responsible for suffusion is explored and the length scales associated with grain detachment and grain transport processes are quantified.

The micromechanical analysis performed in this paper uses a discrete element method [3] implemented in YADE software [10]. Within this framework, a soil sample is modeled as a poly-disperse assembly of rigid spheres in interaction through the classical elasto-frictional contact law implemented in YADE [10] with a Young modulus of $325 \mathrm{MPa}$, a stiffness ratio of 0.42 and a friction angle of $35^{\circ}$.

Bearing in mind that suffusion involves the transport of the smallest particles of a soil, cubic assemblies of spheres are generated randomly with a uniform radius distribution between $r_{\min }=3.6 \times 10^{-5} \mathrm{~m}$ and $r_{\max }=10 r_{\min }$ such that the smallest particles are likely to be transported through the voids created by the largest ones according to the Therzaghi filter rule: $r_{\min } \ll 4 r_{\max }$.

Based on this grading, a dense cubic assembly of 10,000 particles with a void index of 0.6 is generated thanks to the radius expansion technique [5]. Then this initially isotropic sample is subjected to a drained triaxial test consisting under a confining pressure of $100 \mathrm{kPa}$ up to a vertical strain $\left|\varepsilon_{z z}\right|=20 \%$.

\section{Grain detachment analysis}

The microscale analysis of the grain detachment mechanism is underpinned by the idea that the most detachable grains should also be the least stressed. As a result, the grains of the sample presented in the previous section are divided into two groups based on the force chain definition introduced in [6] according to on the following three characteristics:

- Chained particles have a higher principal stress than the mean particle principal stress;

- Their principal stress direction is aligned with the geometrical contact direction (less than $45^{\circ}$ deviation);

- Force chains are composed of at least three contacting particles.

Based on this definition, force chains can be identified for several strain values. A typical visualization of the force chains can be seen in Figure 1 in the initial and critical states. At the beginning of the triaxial test, the force chains are distributed in an isotropic way which is consistent with the fact that no principal direction of loading exists. Once the deviatoric loading is applied, the force chains tend to align in the vertical direction of the macroscopic principal stress.

In the literature, suffusion susceptibility is often considered based on a partition between fine and coarse particles according to a radius threshold. However, the ability of an internal flow to detach some particles should be preferentially linked to the 


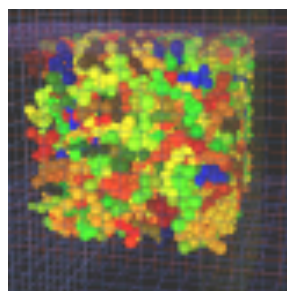

(a) $\varepsilon_{z z}=0 \%$

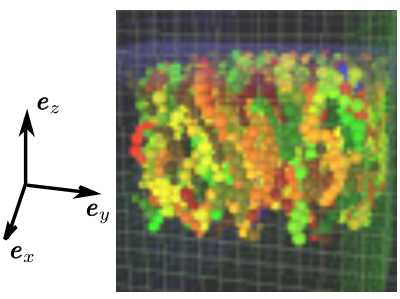

(b) $\varepsilon_{z z}=20 \%$

Fig. 1 Force chains visualisation under an isotropic confining pressure of $100 \mathrm{kPa}$ (a) and at the end of the triaxial test (b).

loading state of the grains. This can be assessed by considering the probability density that a particle of a given radius belongs to a force chain which is illustrated in Figure 2 in the initial configuration of Figure 1.

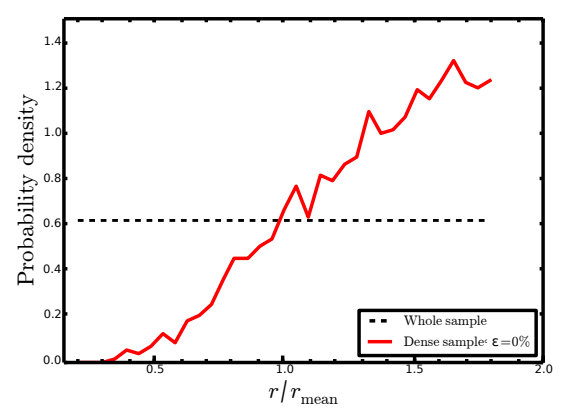

(a)

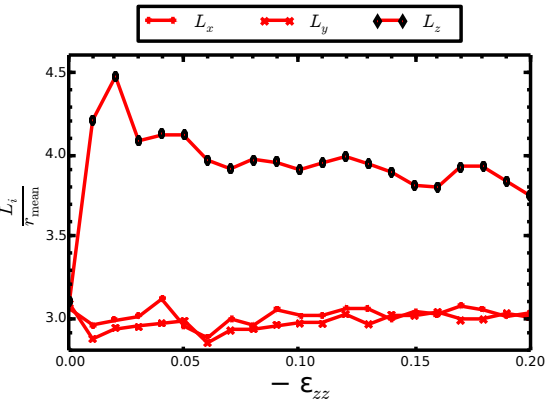

(b)

Fig. 2 Probability density for a grain of a given radius to be part of a force chain at $\varepsilon_{z z}=0 \%$ (a). Strain evolution of the autocorrelation lengths during the triaxial test (b).

Figure 2 shows that force chains are mainly composed of large particles. The dual comment is that the finest particles are the least loaded ones and therefore the most likely to be detached, provided that fluid forces are large enough. Fine particles are also good candidates for being transported over large distances, which will be estimated in section 3. However, no sharp transition is visible in the probability densities in Figure 2. As a result, no clear radius threshold exists between the loose particles of a granular assembly and the primary fabric responsible for stress transmission which is consistent with recent findings [8].

If the previous result gives some information about the geometrical characteristics of the chained particles, it does not give any information on the spatial distribution of force chains. By considering the two-point spatial autocorrelation function between the chained particles, a length scale associated with the detachment process can be introduced as a typical distance between grains transmitting stresses through the 
granular assembly. The two-point spatial autocorrelation function $C$ is defined for any vector $h=\left(h_{x}, h_{y}, h_{z}\right)$ as the joint probability that a point $x$ and the translated point $x+h$ simultaneously belong to the domain $\Omega$ occupied by chained particles:

$$
C:\left\{\begin{array}{l}
\mathbb{R}^{3} \mapsto \mathbb{R} \\
h \mapsto \mathbb{P}\{x \in \Omega \cap x+h \in \Omega\}
\end{array} .\right.
$$

This autocorrelation function can be written in a dimensionless form as

$$
\begin{aligned}
& \tilde{C}(h)=\frac{C(h)-C(0)^{2}}{C(0)-C(0)^{2}} \text { and approximated as } \\
& \tilde{C}_{\mathrm{fit}}(h)=\exp \left(-2 \pi^{1 / 3} \sqrt{\left(\frac{h_{x}}{L_{x}}\right)^{2}+\left(\frac{h_{y}}{L_{y}}\right)^{2}+\left(\frac{h_{z}}{L_{z}}\right)^{2}}\right) .
\end{aligned}
$$

In Equation 2, three length scales $L_{x}, L_{y}$ and $L_{z}$ are introduced to quantify the decrease rate of $\tilde{C}$ and define a virtual unit cell in which the microstructure is highly correlated with respect to stress transmission. The strain evolution of these three autocorrelation lengths is plotted in Figure 2 during the triaxial loading introduced in section 1 . As the horizontal autocorrelation lengths remains constant around $3 r_{\text {mean }}$, the vertical autocorrelation length increases up to a peak value of $4.5 r_{\text {mean }}$ before decreasing and stabilizing around $4 r_{\text {mean }}$. The increase in the vertical autocorrelation corresponds to a lengthening of the force chains along the vertical direction which accounts for the initial hardening of our dense sample. The following decrease in the vertical autocorrelation accounts for the destruction of force chains and thus to the stress softening observed in the end of the triaxial test.

\section{Grain transport analysis}

In order to understand the grain transport mechanism from a micromechanical point of view, a description of the void phase based on the previous work of [7] and [9] is proposed in this section. The description of the void space is reduced to an oriented graph composed of pores (the nodes of the graph) and constrictions (the edges of the graph) based on the tessellation of the granular assembly. Pore centers are defined at the center of each tetrahedron of a regular Delaunay triangulation and pore radii as the radius of the largest interior sphere in the associated tetrahedra. The constrictions are modeled as cylinders joining two adjacent pores and their radii are defined as the radius of the largest interior circle on the common face of the two tetrahedra defining the constriction.

Based on this description of the pore space, the probability density for a free particle of a given radius to find a constriction large enough to travel from one pore to an other can be computed. This probability density is shown in Figure 3 together with the probability density corresponding to the radius distribution of the non-chained grains identified in the previous section.

The comparison between the constriction probability density and the grain probability density in Figure 3 highlights that a large number of grains are small enough to be transported through the pore network. An even larger fraction of the non-chained 


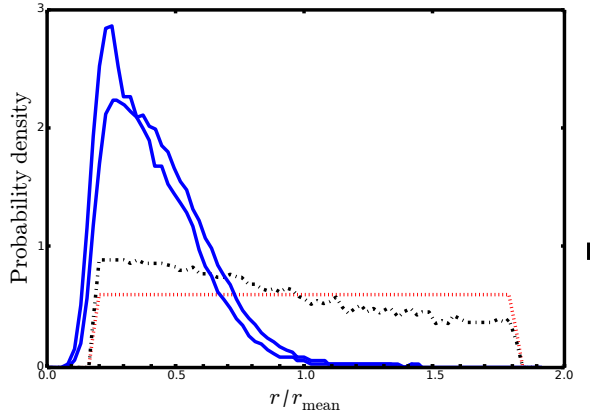

(a)

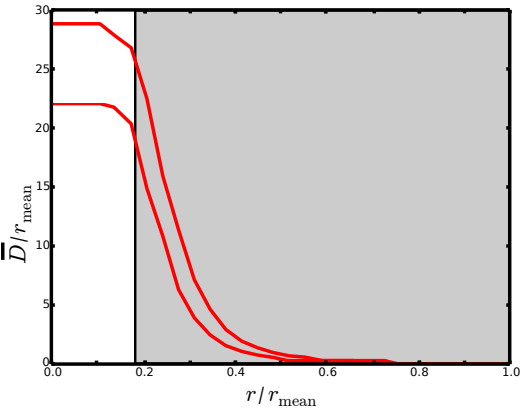

(b)

Fig. 3 Probability density for a constriction (solid), a grain (dotted) or a non-chained grain (dashdotted) to be of a given radius (a). Mean travel distance for different radius thresholds (b). The thick line corresponds to $\left|\varepsilon_{z z}\right|=20 \%$, while the thin line to $\left|\varepsilon_{z z}\right|=0 \%$. The grey domain in the background corresponds to the radius values for the grains composing the sample.

grains is concerned as 33 to $34 \%$ of these grains have a radius smaller than the mean constriction radius plus one standard deviation.

Even though many particles are identified in Figure 3 as potentially transportable by analyzing the statistical properties of the pore network, determining the distance that a particle of a given radius can cover requires knowing the spatial distribution of pores and constrictions together with the fluid flow direction. This can be achieved thanks to the combined used of the DEM-PFV model implemented in YADE [2] together with the definition of transport paths based on a propagation criterion:

- First, the DEM-PFV scheme is used to generate a pressure map in the pore network under a horizontal fluid flow imposed by a small pressure drop of $10 \mathrm{~Pa}$;

- Then, based on the Hagen-Poiseuille flow velocity profile for a circular tube and the expression of the drag force of a uniform flow acting on a sphere for low Reynolds numbers, a flow intensity indicator is defined in each constriction as $\frac{\Delta p}{\ell} \times R^{2}$ with $\ell$ being the length of the constriction, $\Delta p$ the pressure drop between the two adjoining pores and $R$ the constriction radius;

- Finally, a transport path is defined for a given particle of radius $r$ and for a given starting pore as the path of maximum flow intensity on the restriction of the pore network to pores and constrictions of radii larger than $r$.

For a given radius $r$, a mean travel distance $\bar{D}(r)$ is computed from statistical averaging of the length of all the existing transport path defined above. This quantity stands as a measure of the typical length associated with grain transport mechanism and is plotted in Figure 3.

The mean travel distances decreases from $22-29 r_{\text {mean }}$ to zero. If no transport is possible for particles larger than roughly $0.5 r_{\text {mean }}$ the smallest particles of the considered samples have good chances to be transported over large distances going up to $25 r_{\text {mean }}$. Indeed, particles smaller than $0.5 r_{\text {mean }}$ represents $25 \%$ of the non-chained grains. 


\section{Conclusion}

In this paper, specific micromechanical tools are developed to investigate the susceptibility of a polydisperse assembly of spherical particles to grain detachment and grain transport. By simultaneously considering the size distributions of the nonchained particles and the constrictions of the pore network, the ability of a fluid flow to modify the microstructure of the samples is analyzed with respect to the fraction of both potentially detachable and transportable particles. For the particular grading used in this study the typical length scale associated with grain transport can be up to ten times larger than that associated with grain detachment. As a result, a scale separation should exist between these two processes for the smallest particles. Eventually, some fully coupled DEM-PFV numerical simulations were run. The numerical results were used to demonstrate the relevance of the developed micromechanical tools and validate the introduced length scales associated with grain detachment and grain transport. These numerical simulations also enable to track the microstructure evolution resulting from force chains rearrangements and grain transport which will pave the way for the micromechanical analysis of the internal fluid flow impact on the bifurcation domain of the considered sample.

\section{References}

1. S. Bonelli. Erosion of geomaterials. John Wiley \& Sons, 2012.

2. B. Chareyre, A. Cortis, E. Catalano, and E. Barthélemy. Pore-scale modeling of viscous flow and induced forces in dense sphere packings. Transport in porous media, 94(2):595-615, 2012.

3. P. A. Cundall and O. D. Strack. A discrete numerical model for granular assemblies. Geotechnique, 29(1):47-65, 1979.

4. T. Kanit, S. Forest, I. Galliet, V. Mounoury, and D. Jeulin. Determination of the size of the representative volume element for random composites: statistical and numerical approach. International Journal of solids and structures, 40(13):3647-3679, 2003.

5. B. D. Lubachevsky and F. H. Stillinger. Geometric properties of random disk packings. Journal of statistical Physics, 60(5-6):561-583, 1990.

6. J. F. Peters, M. Muthuswamy, J. Wibowo, and A. Tordesillas. Characterization of force chains in granular material. Physical review E, 72(4):041307, 2005.

7. N. Reboul, E. Vincens, and B. Cambou. A statistical analysis of void size distribution in a simulated narrowly graded packing of spheres. Granular Matter, 10(6):457-468, 2008.

8. H. D. To, S. A. G. Torres, and A. Scheuermann. Primary fabric fraction analysis of granular soils. Acta Geotechnica, 10(3):375-387, 2015.

9. E. Vincens, K. J. Witt, and U. Homberg. Approaches to determine the constriction size distribution for understanding filtration phenomena in granular materials. Acta Geotechnica, 10(3):291-303, 2015.

10. V. Šmilauer et al. Yade Documentation 2nd ed. The Yade Project, 2015. http://yadedem.org/doc/. 